\title{
Out-patient psychological therapies for adults with anorexia nervosa: randomised controlled trial
}

Ulrike Schmidt, Anna Oldershaw, Fatima Jichi, Lot Sternheim, Helen Startup, Virginia McIntosh, Jennifer Jordan, Kate Tchanturia, Geoffrey Wolff, Michael Rooney, Sabine Landau* and Janet Treasure*

\section{Background}

Very limited evidence is available on how to treat adults with anorexia nervosa and treatment outcomes are poor. Novel treatment approaches are urgently needed.

\begin{abstract}
Aims
To evaluate the efficacy and acceptability of a novel psychological therapy for anorexia nervosa (Maudsley Model of Anorexia Nervosa Treatment for Adults, MANTRA) compared with specialist supportive clinical management (SSCM) in a randomised controlled trial.
\end{abstract}

\section{Method}

Seventy-two adult out-patients with anorexia nervosa or eating disorder not otherwise specified were recruited from a specialist eating disorder service in the UK. Participants were randomly allocated to 20 once weekly sessions of MANTRA or SSCM and optional additional sessions depending on severity and clinical need (trial registration: ISRCTN62920529). The primary outcomes were body mass index, weight and global score on the Eating Disorders Examination at end of treatment (6 months) and follow-up (12 months). Secondary outcomes included: depression, anxiety and clinical impairment; neuropsychological outcomes; recovery rates; and additional service utilisation.

\section{Results}

At baseline, patients randomised to MANTRA were significantly less likely to be in a partner relationship than those receiving SSCM $(3 / 34$ V. 10/36; $P<0.05)$. Patients in both treatments improved significantly in terms of eating disorder and other outcomes, with no differences between groups. Strictly defined recovery rates were low. However, MANTRA patients were significantly more likely to require additional in-patient or day-care treatment than those receiving SSCM (7/34 v. 0/37; $P=0.004)$.

\section{Conclusions}

Adults with anorexia nervosa are a difficult to treat group. The imbalance between groups in partner relationships may explain differences in service utilisation favouring SSCM. This study confirms SSCM as a useful treatment for out-patients with anorexia nervosa. The novel treatment, MANTRA, designed for this patient group may need adaptations to fully exploit its potential.

\section{Declaration of interest}

U.S. receives salary support from the NIHR (Mental Health Biomedical Research Centre) at South London and Maudsley NHS Foundation Trust and King's College London.
Anorexia nervosa is a mental disorder with high levels of chronicity, mortality and burden on families. ${ }^{1-3}$ Treatment is difficult, in part due to its ego-syntonic nature. Anxious and obsessional personality traits are common and negatively impact outcome. ${ }^{1}$ Moreover, people with anorexia have trait-like impairments in neurocognitive and social functioning, with impaired set-shifting (cognitive rigidity), poor central coherence (excessive detail focus at the expense of the bigger picture) and impairments in the processing of socio-emotional stimuli. ${ }^{4-6}$

Psychotherapy is the treatment of choice for anorexia nervosa, ${ }^{7}$ however in adults (i.e. those with a more chronic illness) outcomes are poor and drop-out is high. ${ }^{8}$ Few trials ${ }^{9-16}$ have tested psychological therapies such as cognitive-behavioural therapy (CBT), cognitive-analytical therapy (CAT), interpersonal therapy (IPT) and family therapy as first-line treatments in adults with anorexia, but no front-runner has emerged. Trials are small and underpowered and have other weaknesses. One more recent trial found specialist psychotherapies to be superior to nonspecialist treatment in adults with anorexia nervosa in terms of weight gain and overall recovery. ${ }^{15}$ Another trial ${ }^{16}$ found specialist supportive clinical management (SSCM) superior on several outcomes compared with CBT and IPT, although in the longer term these therapies were indistinguishable. ${ }^{17}$ This suggests that specialist knowledge and skills are important in the treatment of adults with anorexia.
In summary, the need to develop more effective treatments for adults with anorexia and to study these in well-conducted adequately powered studies remains pressing. ${ }^{7}$

We have developed a specific maintenance model and treatment of anorexia nervosa, ${ }^{18}$ the Maudsley Model of Anorexia Nervosa Treatment for Adults (MANTRA). This is novel in several respects: (a) it is biologically informed and trait-focused, drawing on neuropsychological, social cognitive and personality trait research; (b) it includes both intra- and interpersonal maintaining factors and strategies to address these; and (c) it is modularised with a hierarchy of procedures, tailored to the individual. A pilot series of 28 adults with anorexia nervosa had good treatment uptake, retention and outcomes. ${ }^{19}$

The present study tested the efficacy and acceptability of MANTRA $v$. SSCM in adult out-patients with anorexia nervosa in a preliminary randomised controlled trial (RCT). We hypothesised that: (a) MANTRA would be more effective than SSCM at end of treatment and follow-up in terms of weight gain and reduction in eating disorder symptoms; and (b) on neuropsychological tasks assessing cognitive rigidity, patients receiving MANTRA would show greater improvements than those receiving SSCM.

\section{Method}

\section{Setting}

The study was conducted in the Eating Disorders Outpatient Service of the South London and Maudsley NHS Foundation Trust. Patients were referred by their general practitioners. 


\section{Participants}

Inclusion criteria

Consecutive referrals were offered participation if they were (a) aged 18 or over, and (b) had DSM-IV ${ }^{20}$ anorexia nervosa or eating disorder not otherwise specified (EDNOS), with a body mass index (BMI) of $<18.5 \mathrm{~kg} / \mathrm{m}^{2}$.

\section{Exclusion criteria}

We excluded patients with life-threatening anorexia nervosa requiring immediate in-patient treatment, ${ }^{7}$ those with insufficient knowledge of English to understand the treatment, intellectual disability, severe mental or physical illness needing treatment in its own right (e.g. psychosis or diabetes mellitus), substance dependence or pregnancy. We did not exclude patients on a stable dose of an antidepressant (i.e. for $\geqslant 4$ weeks).

\section{Recruitment}

Recruitment took place between March 2007 and February 2009. Informed written consent was sought from participants at assessment after complete description of the study. The local ethics committee approved the study (ethics committee number: 06/Q0706/105).

\section{Design}

This was a two-arm RCT (trial registration: ISRCTN62920529). We considered including a no treatment control group, but given the life-threatening nature of anorexia nervosa believed this to be unethical, as patients who are allocated to waiting list or assessment only either do not improve ${ }^{21}$ or seek treatment elsewhere. $^{11}$

\section{Interventions}

\section{commonalities between both treatments}

In both treatments, patients received 20 once-weekly individual sessions of therapy and 4 monthly follow-up sessions. Two additional sessions with a close other were offered, as were assessment from the team's dietician with follow-up as needed. In low-weight patients $\left(\mathrm{BMI} \leqslant 15 \mathrm{~kg} / \mathrm{m}^{2}\right)$, treatment could be extended to 30 weekly sessions plus 4 follow-ups.

\section{MANTRA}

The model underpinning this treatment proposes that anorexia nervosa typically arises in people with anxious/obsessional traits. Core to the maintenance of anorexia are four broad factors. ${ }^{18}$ First, an information-processing style characterised by cognitive rigidity and attention to detail at the expense of the bigger picture. Second, impairments in the socio-emotional domain (such as avoidance of the experience and expression of emotions in the context of close relationships). Starvation intensifies these problems. Third, consonant with these impairments, these individuals typically develop beliefs about the utility of anorexia nervosa in their lives. ${ }^{22}$ Fourth, parents or partners may inadvertently maintain the disorder by high levels of expressed emotion $^{23}$ or by accommodation and enabling behaviours. ${ }^{24}$

The MANTRA model is centred around a patient workbook (online Table DS1), with patient and therapist deciding collaboratively which parts might be relevant. The therapy style is that of motivational interviewing. ${ }^{25}$ The principles of behavioural change are used to guide patients towards recovery. ${ }^{26}$

There are several treatment phases. In the initial phase, a dialogue about change is developed by (a) giving feedback about the person's health risk and their cognitive style based on neurocognitive tests, and (b) an exploration of the valued function of anorexia in the person's life, aiming to shift the balance between positive and negative illness beliefs to create an impetus for change. In the formulation phase, a collaborative case conceptualisation and treatment plan are developed and presented to the patient as a letter and a diagram. In the working for change phase, modules focusing on socio-emotional impairments and/or thinking style are introduced as appropriate. Behavioural experiments focused on reducing these impairments are conducted. For those with chronic anorexia, a module on developing an identity beyond anorexia is available. The final phase of treatment concentrates on relapse prevention and ending.

\section{SSCM}

This treatment was developed as a comparison treatment to CBT and IPT for an RCT in out-patients with anorexia. ${ }^{16}$ Specialist supportive clinical management is designed to be delivered by eating disorder specialists and aims 'to mimic outpatient treatment that could be offered to individuals with anorexia nervosa in usual clinical practice. ${ }^{16}$ It combines elements of clinical management and supportive psychotherapy. The abnormal nutritional status and dietary patterns of anorexia are central to SSCM, which emphasises the resumption of normal eating and restoration of weight, and provides information on weight gain and weight maintenance strategies, energy requirements and re-learning to eat normally. Other therapy content is dictated by the patient. Further details of this treatment are described elsewhere. ${ }^{27}$ There is a manual for therapists (available from the authors on request) with psychoeducational handouts for patients. Similarities and differences between MANTRA and SSCM are shown in online Table DS2.

Treatments were delivered by 16 experienced eating disorder therapists. Therapists participated in training workshops for both therapies prior to the study and received weekly supervision. The SSCM workshop was run by V.M., one of the developers of this treatment. To protect against contamination between therapies, therapists were randomly allocated to deliver one of the two therapies only, stratifying for therapist experience and training.

\section{Treatment fidelity}

Two experienced supervisors provided weekly supervision, separately for MANTRA and SSCM therapists. Therapy sessions were audio-taped to allow analysis of the therapeutic process (to be reported separately). V.M. and J.J. were available for advice on SSCM.

\section{Management of serious risk}

Patients who deteriorated significantly while receiving out-patient therapy were offered in-patient treatment if they fulfilled criteria for admission. ${ }^{7}$ Those who failed to improve with out-patient treatment were offered day care.

\section{Assessments}

An initial structured clinical interview determined patients' eligibility for the study. Research assessments took place at baseline, 6 months and 12 months.

\section{Eating disorder}

The patient's height was measured at initial assessment and weight was measured at each assessment. The BMI was calculated. 
Eating Disorder Examination and Clinical Impairment Assessment Questionnaire. The Eating Disorder Examination $(\mathrm{EDE})^{28}$ is a semi-structured interview for assessing eating disorder symptomatology. We used this (a) at baseline to make DSM diagnoses, and (b) to assess eating disorder symptoms over the previous month at baseline, 6 months and 12 months. The Clinical Impairment Assessment Questionnaire (CIA) ${ }^{29}$ is a 22-item self-report measure of the severity of psychosocial impairment due to eating disorder features over the past 28 days.

\section{Questionnaire-based measures of other psychopathology}

Comorbid psychopathology was assessed using the Hospital Anxiety and Depression Scale (HADS). ${ }^{30}$

\section{Neurocognitive measures}

As MANTRA targets cognitive inflexibility, three set-shifting tasks were administered: Brixton Spatial Anticipation Task (BSAT), ${ }^{31}$ Wisconsin Card Sorting Task (WCST) ${ }^{32}$ and Trail Making Task (TMT). ${ }^{33}$

\section{Service utilisation}

No formal assessment of service utilisation was conducted. Information about additional intensive eating disorder treatment (in-patient or day-care treatment) was obtained from patients' case notes.

\section{Other measures}

Treatment expectation and satisfaction were assessed using visual analogue scales at the beginning and end of treatment respectively. A range of additional measures assessed other aspects of the MANTRA model. These are reported separately.

\section{Sample size}

Calculations were based on the mean weight gain $(7 \mathrm{~kg})$ of an unpublished series of ten patients treated with MANTRA and mean weight gain for SSCM $(4 \mathrm{~kg})$ described in McIntosh et al. ${ }^{16}$ A sample size of 29 per group would have $80 \%$ power to detect a difference in mean weight gain of $3 \mathrm{~kg}$, assuming a common standard deviation of $4 \mathrm{~kg}$ using a two group $t$-test with 0.05 two-sided significance level. A drop-out rate of $15 \%$ was allowed for, increasing the required sample size to 35 per group.

\section{Randomisation, masking and protection against bias}

Patients were randomised after baseline assessment. A researcher independent from the trial team generated the randomisation codes using a computerised system. Randomisation was stratified by eating disorder severity (BMI above or below $15 \mathrm{~kg} / \mathrm{m}^{2}$ ). Treatment assignment codes were contained in numbered sealed opaque envelopes held by the independent researcher. An administrator was notified of treatment allocation and then sent a letter to inform the patient.

Outcome assessments were conducted by two assessors masked to treatment allocation. Participants were reminded at the beginning of each assessment not to reveal their treatment allocation to the assessor. To test masking success, assessors were asked to guess the treatment group of the participant after the end of each participant's participation in the study. Treatment allocation was guessed correctly in $53.5 \%$ of cases, i.e. masking was successful.

\section{Statistical analysis}

The purpose of the statistical analyses was to compare outcome variables between the two treatment arms, MANTRA and SSCM. All statistical analyses were performed in Stata version 11 for Windows XP, following the intention-to-treat principle.

The primary outcomes were BMI score, weight and EDE global score at months 6 and 12. The secondary outcomes were HADS depression score, HADS anxiety score, CIA score, BSAT score, WCST score and TMT time at months 6 and 12 .

A linear mixed model was used to evaluate the effect of treatment arm on the different outcomes. Each outcome was analysed by a separate mixed effects model. The outcome measures at 6 and 12 months formed the dependent variable. The fixed part of the model included: a treatment dummy variable (SSCM/MANTRA) to account for the difference between the two arms at 6 months; a time dummy variable ( 6 months/ 12 months) to allow for change over time; and a treatment $\times$ time interaction to assess the change in the group difference over time. The prerandomisation (baseline) measure of the outcome was included as a covariate as was the stratification factor baseline BMI. Further baseline variables hypothesised to be predictive of outcome (length of illness) were tested and only included if they were found to be predictive. The random part of the model included participant-varying intercepts. Therapist identity was also explored as a further random intercept to allow for therapist clustering. However, likelihood ratio tests comparing the model with random effects at the level of therapist and participant to the model with just participant identity as a random effect were performed using complete case analysis and did not detect any benefit of including therapist effects in the model at the 5\% level $(P=1.00)$. Thus, only individual random intercepts were retained.

Linear mixed modelling of the available data gives valid estimators, provided that data are missing at random and the observable variables predicting missingness are included in the analysis model. We empirically assessed a number of baseline variables (age, education, occupation, length of illness duration) and post-treatment variables (number of treatment sessions) for their ability to predict missingness at each time point measured by a binary variable (missing data at that time point for that participant Yes/No) using logistic regression.

Baseline variables that were predictive of missingness at the $5 \%$ significance level were included in the linear mixed model as further covariates. We found that the post-treatment variable 'number of treatment sessions' also predicted missingness, with the probability of loss to follow-up increasing with decreasing number of sessions attended. Therefore, to provide valid inferences under this form of missing at random, we employed multiple imputation ${ }^{34}$ which allowed us to use 'number of sessions' during the imputation step without having to include this post-treatment variable in the analysis model. All primary and secondary outcome variables measured at baseline, 6 months or 12 months, treatment arm and length of illness duration as well as 'number of treatment sessions' were included in the imputation step. Two hundred imputations were run. Multiple imputation was carried out in Stata using the ice and mim commands. ${ }^{35}$

As a sensitivity analysis, a treatment complier analysis was carried out using only individuals who completed 14 or more sessions.

\section{Results}

\section{Patient flow and service utilisation}

Figure 1 shows the participant flow through the study: 34 participants were randomised to MANTRA and 37 to SSCM. Treatment uptake was comparable between groups. 


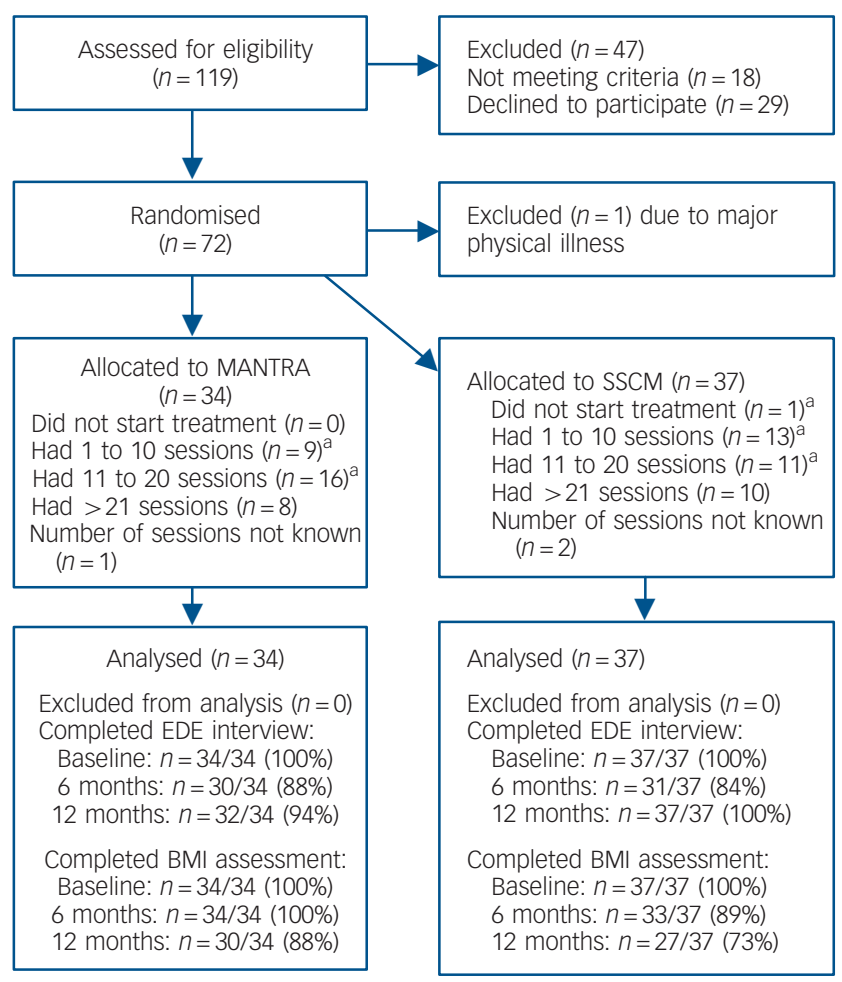

Fig. 1 CONSORT diagram

BMI, body mass index; EDE, Eating Disorders Examination; MANTRA, Maudsley Model of Anorexia Nervosa Treatment for Adults; SSCM, specialist supportive clinical
management.

a. We did not formally record the reasons for non-completion. For the majority of these cases this was simply that they decided to leave treatment prematurely. A small number moved away or had a lower than planned number of sessions by agreement with their therapist.

Treatment expectations were comparable and satisfaction was high with no differences between groups. Details can be obtained from the authors. The mean number of sessions attended in MANTRA was 14.4 (s.d.=7.0) and in SSCM was 14.2 (s.d.=9.5). Seven patients required hospitalisation or day-care treatment during the trial; all had been allocated to MANTRA. This difference was significant (Fisher's exact test 0.004). No patient died.

Groups did not differ in terms of the proportions of people who had additional sessions with the dietician (MANTRA 17/34 (50\%); SSCM 14/37 (38\%); $\chi^{2}=1.065$; d.f. $\left.=1 ; P=0.302\right)$. There was a trend for MANTRA patients to have more dietician sessions (median $=0.5$; interquartile range $(\mathrm{IQR})=3$ ) than SSCM patients (median $=0.0 ; \mathrm{IQR}=1$; Mann-Whitney $U$-test $P=0.08$ ). This was explained by a significantly higher number of dietician sessions (median $=3.6 ; \mathrm{IQR}=4$ ) of patients who needed additional in-patient or day-care treatment compared with the rest of the MANTRA group (median $=0 ; \mathrm{IQR}=1 ;$ Mann-Whitney $U$-test $P=0.015)$.

\section{Patient characteristics at baseline}

Patients in the two treatment groups were similar in terms of baseline sociodemographic and clinical characteristics (Table 1), with the exception of relationship status where more people allocated to SSCM were in a relationship than those allocated to MANTRA $\left(\chi^{2}(1)=4.2, P<0.05\right)$.

\section{Treatment outcomes}

Length of illness duration was not predictive of outcomes $(P>0.980)$ and was not included in the fixed parts of the models.
We present estimated treatment effects on primary and secondary outcomes at both post-treatment time points (with baseline outcome values and baseline BMI held constant) to assess the efficacy of MANTRA relative to SSCM (Tables 2 and 3) as well as estimated differences between post-treatment outcome group means and the baseline sample average to examine change over time after receiving any treatment (Tables 4 and 5).

\section{Primary outcomes}

Tables 2 and 3 show that there was no difference in the effect of treatments at either time point for BMI, weight or EDE Global score (all $P>0.5$ ).

As Tables 4 and 5 show, there was, however, a significant effect of receiving treatment, with mean $\mathrm{BMI}$ increasing from baseline to month 6 by 0.88 ( $95 \%$ CI $0.38-1.39$ ), and from baseline to month 12 by 1.26 (95\% CI 0.41-2.12). This is illustrated in Fig. 2.

The mean increase in weight from baseline to month 6 was $2.49 \mathrm{~kg}$ (95\% CI 1.17-3.82) and from baseline to month 12 was $3.55 \mathrm{~kg}$ (95\% CI 2.07-5.03); showing a significant effect of receiving either treatment (Fig. 3).

There was also an effect of being given treatment on EDE Global score, with mean EDE decreasing from baseline to month 6 by 1.02 (95\% CI 1.45-0.59) and from baseline to month 12 by 1.28 (95\% CI 2.03-0.54) (Fig. 4).

The complier analysis produced similar results to those presented and did not change any conclusions.

\section{Secondary outcomes}

Tables 2 and 3 show that there was no evidence of a difference between the two treatment groups at either month 6 or 12 for any secondary outcome (all $P>0.4$ ).

The HADS and CIA scores showed significant improvements after treatment, whereas BSAT, WCST and TMT outcomes did not change significantly (all $P>0.05$ ) (Tables 4 and 5).

\section{Recovery rates}

These were defined in three ways: (a) as the proportion of patients with an EDE Global score of less than 1 standard deviation above community mean (i.e. below 1.74$) ;^{36}$ (b) as the proportion of patients with a BMI $>18.5 \mathrm{~kg} / \mathrm{m}^{2}$ (using the World Health Organization's cut-off for normal weight); and (c) as the proportion of patients with a normal EDE (as above) and a BMI $>18.5 \mathrm{~kg} / \mathrm{m}^{2}$. Proportions of people with normal EDE at the three time points (baseline, 6 and 12 months) were as follows: MANTRA 5/34 (15\%), 13/30 (43\%) and 19/32 (59\%) respectively; SSCM 5/37 (14\%), 14/31 (45\%) and 27/37 (73\%) respectively. Proportions of people with a BMI $>18.5 \mathrm{~kg} / \mathrm{m}^{2}$ at the three time points were as follows: MANTRA $0(0 \%), 6 / 34(18 \%)$ and $8 / 30$ (27\%); SSCM 0 (0\%), 7/33 (21\%) and 7/27 (26\%). Proportions of people with normal EDE and a BMI $>18.5 \mathrm{~kg} / \mathrm{m}^{2}$ at the three time points were as follows: MANTRA $0(0 \%), 2 / 30(7 \%)$ and 4/29 (14\%); SSCM $0(0 \%), 4 / 30(13 \%)$ and 5/27 (19\%). There were no significant differences between the two groups $\left(\chi^{2}\right.$ or Fisher's exact tests; $P$-values between 0.23 and 0.94 ).

\section{Moderator analysis}

An exploratory moderator analysis of patients with higher BMI at baseline (between 17.5 and 18.5; $n=18$ : MANTRA $n=9$, SSCM $n=9$ ) and lower BMI (below 17.5; $n=53$ : MANTRA $n=25$, SSCM $n=28$ ) at baseline, looking at 12 month outcomes (weight, EDE Global score, CIA, HADS, BSAT, WCST and TMT). In patients with higher initial BMI there was no evidence of a moderation effect with $P$-values between 0.368 and 0.953 . In patients with 


\begin{tabular}{|c|c|c|c|c|c|c|}
\hline & \multicolumn{2}{|c|}{ Whole group } & \multicolumn{2}{|c|}{ MANTRA } & \multicolumn{2}{|c|}{ SSCM } \\
\hline & N & & $N$ & & N & \\
\hline \multicolumn{7}{|l|}{ Demographic details } \\
\hline Age, years: mean (s.d.) & 70 & $26.6(7.9)$ & 34 & $25.6(6.9)$ & 36 & $27.5(8.7)$ \\
\hline Males:females, $n$ & 71 & $5: 66$ & 34 & $3: 31$ & 37 & $2: 35$ \\
\hline Ethnicity, $n(\%)$ & 71 & & 34 & & 37 & \\
\hline White British & & $52(73.2)$ & & $26(76.5)$ & & $26(70.3)$ \\
\hline White Other & & $5(7.0)$ & & $3(8.8)$ & & $2(5.4)$ \\
\hline Black British & & $3(4.2)$ & & 0 & & $3(8.1)$ \\
\hline Black Other & & 0 & & 0 & & 0 \\
\hline Asian British & & $4(5.6)$ & & $2(5.8)$ & & $2(5.4)$ \\
\hline Asian Other & & $4(5.6)$ & & $1(2.9)$ & & $3(8.1)$ \\
\hline Other & & $3(4.2)$ & & $2(5.89)$ & & $1(2.7)$ \\
\hline Years in education, mean (s.d.) & 55 & $14.9(2.8)$ & 27 & $15.0(2.9)$ & 28 & $14.8(2.7)$ \\
\hline In a relationship, $n(\%)$ & 70 & $13(18.3)$ & 34 & $3(8.8)$ & 36 & $10(27)$ \\
\hline \multicolumn{7}{|l|}{ Clinical details } \\
\hline Diagnosis, $n$ (\%) & 71 & & 37 & & 34 & \\
\hline AN-R & & $25(35.2)$ & & $14(41.2)$ & & $11(29.7)$ \\
\hline AN-BP & & $24(33.8)$ & & $11(32.4)$ & & $13(35.1)$ \\
\hline EDNOS-R & & $20(28.2)$ & & $9(26.5)$ & & $11(29.7)$ \\
\hline EDNOS-BP & & $2(2.8)$ & & 0 & & $2(5.4)$ \\
\hline BMI, kg/m²: mean (s.d.) & 71 & $16.4(1.3)$ & 34 & $16.3(1.3)$ & 37 & $16.4(1.3)$ \\
\hline Weight, kg: mean (s.d.) & 71 & $44.3(5.1)$ & 34 & $44.9(5.7)$ & 37 & $43.7(4.5)$ \\
\hline Age at onset, years: mean (s.d.) & 70 & $18.9(6.5)$ & 33 & $19.1(8.1)$ & 37 & $18.7(4.8)$ \\
\hline Duration of illness, months: mean (s.d.) & 58 & $80.6(71.8)$ & 27 & $77.3(70.8)$ & 31 & $83.5(73.6)$ \\
\hline Lowest BMI since onset, $\mathrm{kg} / \mathrm{m}^{2}$ : mean (s.d.) & 54 & $15.2(1.8)$ & 29 & $15.3(1.7)$ & 25 & $15.2(2.0)$ \\
\hline Previous eating disorder treatment, $n$ (\%) & 66 & $42(59.2)$ & 31 & $17(50)$ & 35 & $25(67.6)$ \\
\hline EDE Global score, mean (s.d.) & 71 & $3.2(1.4)$ & 34 & $3.3(1.5)$ & 37 & $3.2(1.3)$ \\
\hline HADS depression score, mean (s.d.) & 71 & $10.3(4.5)$ & 34 & $10.9(4.9)$ & 37 & $9.6(4.2)$ \\
\hline HADS anxiety score, mean (s.d.) & 71 & $13.1(4.2)$ & 34 & $12.6(4.9)$ & 37 & $13.5(3.4)$ \\
\hline CIA, mean (s.d.) & 70 & $1.8(0.7)$ & 34 & $1.8(0.7)$ & 36 & $1.8(0.7)$ \\
\hline Current antidepressant medication, $n$ (\%) & 64 & $24(33.8)$ & 31 & $9(26.5)$ & 33 & $15(40.5)$ \\
\hline
\end{tabular}

\begin{tabular}{|c|c|c|c|c|c|}
\hline \multirow[b]{2}{*}{ Outcome } & \multicolumn{2}{|c|}{$\begin{array}{l}\text { Predicted mean (s.e.), baseline fixed } \\
\text { to sample average }\end{array}$} & \multirow{2}{*}{$\begin{array}{l}\text { Estimated treat- } \\
\text { ment difference }\end{array}$} & \multirow[b]{2}{*}{$95 \% \mathrm{Cl}$} & \multirow[b]{2}{*}{$P$} \\
\hline & MANTRA & SSCM & & & \\
\hline Body mass index & $17.27(0.36)$ & $17.24(0.36)$ & 0.03 & -0.98 to 1.04 & 0.953 \\
\hline Weight & $47.00(0.96)$ & $46.53(0.96)$ & 0.48 & -2.19 to 3.15 & 0.725 \\
\hline EDE Global & $2.09(0.31)$ & $2.33(0.32)$ & -0.24 & -1.13 to 0.65 & 0.596 \\
\hline HADS anxiety & $11.15(1.00)$ & $11.18(0.97)$ & -0.03 & -2.69 to 2.63 & 0.983 \\
\hline HADS depression & $8.00(1.01)$ & $8.16(1.03)$ & -0.165 & -2.99 to 2.66 & 0.909 \\
\hline Clinical Impairment Assessment & $1.30(0.19)$ & $1.40(0.19)$ & -0.10 & -0.63 to 0.42 & 0.699 \\
\hline Brixton Spacial Anticipation Task & $12.79(1.40)$ & $12.34(1.47)$ & 0.45 & -3.51 to 4.41 & 0.822 \\
\hline Wisconsin Card Sorting Task & $16.45(4.05)$ & $12.09(4.57)$ & 4.36 & -7.08 to 15.81 & 0.454 \\
\hline Trail Making Task & $34.38(3.21)$ & $36.57(3.47)$ & -2.19 & -11.41 to 7.04 & 0.642 \\
\hline
\end{tabular}

lower initial BMI too, none of the subgroup tests was significant at the 5\% level, however the effect of treatment favouring MANTRA was strongest on weight (coefficient 3.141 ; $95 \%$ CI -1.23 to 7.51 ; $P=0.159)$.

\section{Discussion}

\section{Main findings}

First, out-patients with anorexia nervosa improved significantly in terms of BMI, weight, eating disorder symptoms, affective symptoms and psychosocial impairment. Second, there were no differences between the two treatment groups. Third, neuropsychological performance did not improve, but baseline levels were not particularly impaired. ${ }^{4}$ Thus, our main hypotheses were not confirmed. These findings deserve comment.

The significant improvement of patients in both groups suggests that both treatments have merit. These improvements are unlikely to be due simply to the passage of time, as previous trials have shown that patients with anorexia do not spontaneously improve without treatment. ${ }^{1,21}$

The lack of difference between the two treatments is disappointing. Overall, the mean weight change for MANTRA 


\begin{tabular}{|c|c|c|c|c|c|}
\hline \multirow[b]{2}{*}{ Outcome } & \multicolumn{2}{|c|}{$\begin{array}{l}\text { Predicted mean (s.e.), baseline fixed } \\
\text { to sample average }\end{array}$} & \multirow{2}{*}{$\begin{array}{l}\text { Estimated treat- } \\
\text { ment difference }\end{array}$} & \multirow[b]{2}{*}{$95 \% \mathrm{Cl}$} & \multirow[b]{2}{*}{$P$} \\
\hline & MANTRA & SSCM & & & \\
\hline Body mass index & $17.77(0.40)$ & $17.62(0.41)$ & 0.15 & -1.00 to 1.29 & 0.801 \\
\hline Weight & $48.13(1.06)$ & 47.51 (1.09) & 0.61 & -2.42 to 3.65 & 0.691 \\
\hline EDE Global & $1.81(0.38)$ & $2.06(0.37)$ & -0.26 & -1.32 to 0.80 & 0.633 \\
\hline HADS anxiety & $10.94(1.27)$ & $10.17(1.48)$ & 0.77 & -2.92 to 4.46 & 0.682 \\
\hline HADS depression & $6.86(1.18)$ & 7.39 (1.34) & -0.53 & -4.00 to 2.95 & 0.765 \\
\hline Clinical Impairment Assessment & $1.16(0.22)$ & $1.16(0.24)$ & 0.0001 & -0.62 to 0.62 & 1.000 \\
\hline Brixton Spacial Anticipation Task & $11.54(1.84)$ & $13.06(2.01)$ & -1.51 & -7.34 to 4.32 & 0.610 \\
\hline Wisconsin Card Sorting Task & $14.28(5.55)$ & $14.03(5.50)$ & 0.40 & -10.73 to 11.53 & 0.944 \\
\hline Trail Making Task & $34.67(3.91)$ & $34.29(4.20)$ & 0.27 & -10.77 to 11.31 & 0.961 \\
\hline
\end{tabular}

was lower than anticipated in our unpublished pilot series on which the power calculation was based. However, SSCM outperformed two active treatments in the only previous trial in which it was used and therefore sets the bar for a control treatment very high. Future trials including SSCM as one of the arms may need to consider being powered for equivalence or non-inferiority.

Patients in this study were not particularly impaired on neuropsychological set-shifting measures, which is of note as participants were relatively unselected patients across the whole severity spectrum of anorexia nervosa. Previous neuropsychological studies have often focused on in-patient or self-selected samples that may have been biased towards greater levels of neuropsychological impairment. As such, the lack of differential improvement on neuropsychological variables is unsurprising.

\section{Other findings}

Retention in the trial was good with comparable adherence, treatment expectations and satisfaction in both groups.

This study explored several definitions of recovery. Defining recovery purely in terms of a normal EDE Global score showed a small proportion of participants already in this category prior to starting, which is unsurprising as we included patients with EDNOS in our trial who did not necessarily have weight and shape

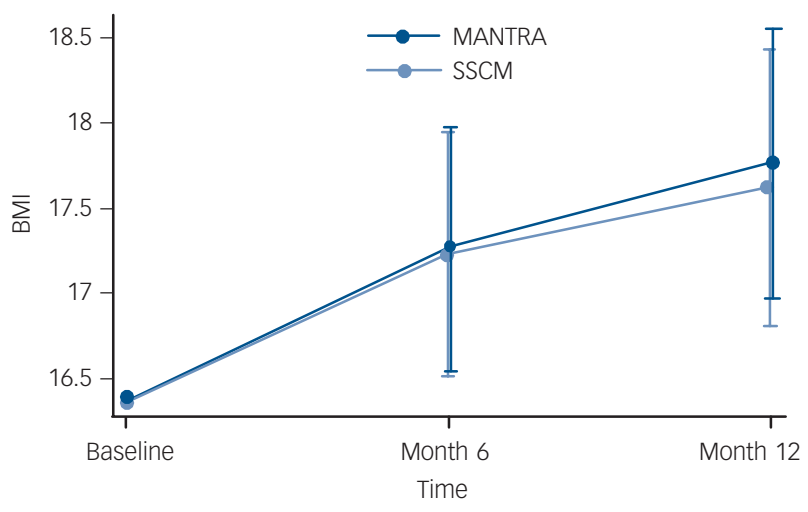

Fig. 2 Predicted mean body mass index (BMI) scores at each time point with $95 \%$ confidence intervals, broken down by treatment group.

MANTRA, Maudsley Model of Anorexia Nervosa Treatment for Adults; SSCM, specialist supportive clinical management. concerns. At 1 year, more than two-thirds of patients had a normal EDE Global score, with no difference between the groups. This suggests that both therapies were very effective in treating broad eating disorder symptomatology. However, much smaller proportions of patients had a normal weight or were recovered combining both criteria at 1 year. This is perhaps not surprising given how unwell some of the patients in the study were to start with. Of note, previous research in adolescents with anorexia similarly reported highly variable recovery rates depending on how this was defined. ${ }^{37}$

Patients receiving MANTRA more frequently required additional treatment. Of note, significantly more SSCM patients were in a partner relationship and this has been shown to predict a more positive outcome in anorexia nervosa. ${ }^{38}$ This imbalance between groups may go some way towards explaining differences in service utilisation favouring SSCM.

\section{Comparison with other trials}

Unlike other studies, the present study included patients along the full spectrum of severity and chronicity of anorexia nervosa. The magnitude of weight improvement is comparable to that found in previous RCTs (e.g. Dare et $a l,{ }^{15}$ McIntosh et $a l^{16}$ ). Treatment gains were achieved with a relatively low number of treatment sessions. The number of patients needing in-patient treatment was low and in contrast to other trials there were no deaths. ${ }^{15,16}$

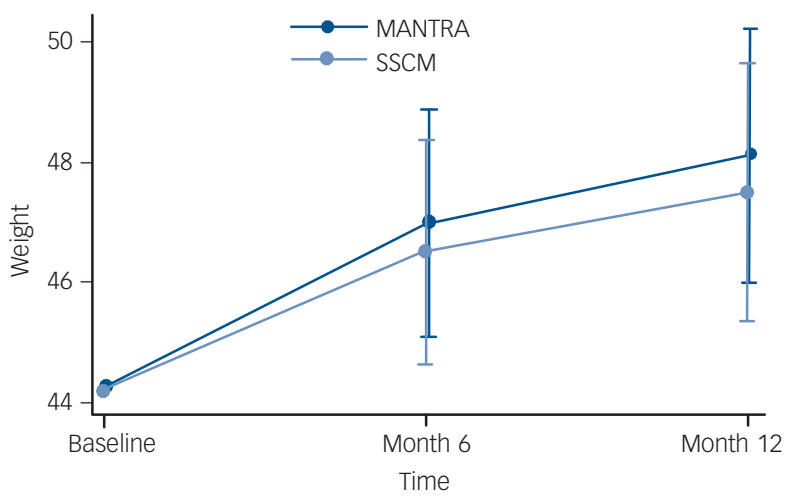

Fig. 3 Predicted mean weight at each time point with $95 \%$ confidence intervals, broken down by treatment group.

MANTRA, Maudsley Model of Anorexia Nervosa Treatment for Adults; SSCM, specialist supportive clinical management. 


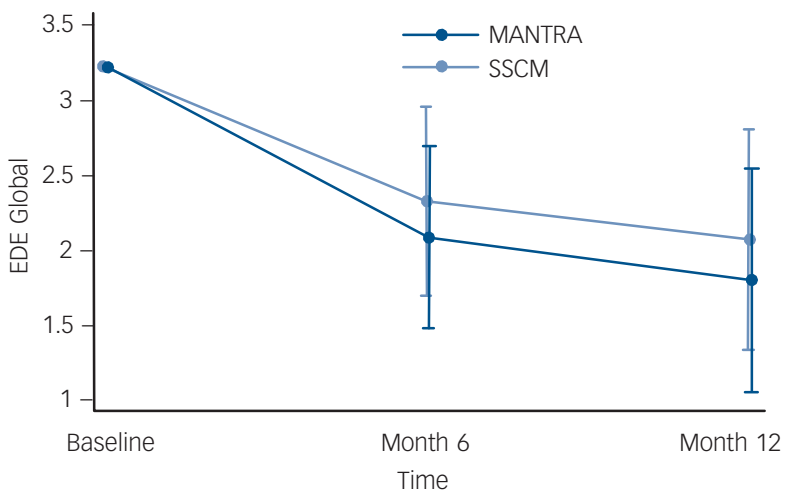

Fig. 4 Predicted mean Eating Disorders Examination (EDE) Global scores at each time point with $95 \%$ confidence intervals, broken down by treatment group.

MANTRA, Maudsley Model of Anorexia Nervosa Treatment for Adults; SSCM, specialist supportive clinical management.

\section{MANTRA and SSCM}

As mentioned above, in the only other trial using SSCM in patients with a mild form of anorexia nervosa, this was superior to two active treatments. ${ }^{16}$ In the present trial, SSCM performed

\begin{tabular}{|c|c|c|c|}
\hline Outcome & Estimate & $95 \% \mathrm{Cl}$ & $P$ \\
\hline \multicolumn{4}{|l|}{ Primary outcomes } \\
\hline Body mass index & 0.88 & 0.38 to 1.39 & 0.001 \\
\hline Weight & 2.49 & 1.17 to 3.82 & $<0.001$ \\
\hline EDE Global & -1.02 & -1.45 to -0.59 & $<0.001$ \\
\hline \multicolumn{4}{|l|}{ Secondary outcomes } \\
\hline HADS anxiety & -1.89 & -3.29 to -0.49 & 0.008 \\
\hline $\begin{array}{l}\text { HADS depression } \\
\text { Clinical Impairment }\end{array}$ & -2.14 & -3.56 to -0.72 & 0.003 \\
\hline $\begin{array}{l}\text { Assessment } \\
\text { Brixton Spacial }\end{array}$ & -0.46 & -0.72 to -0.20 & 0.001 \\
\hline $\begin{array}{l}\text { Anticipation Task } \\
\text { Wisconsin Card Sorting }\end{array}$ & -1.88 & -3.89 to 0.14 & 0.068 \\
\hline Task & -6.19 & -15.42 to 3.06 & 0.188 \\
\hline Trail Making Task & -3.96 & -8.65 to 0.72 & 0.097 \\
\hline
\end{tabular}

Table 5 Estimated change in mean outcome between baseline and month 12 (average of MANTRA and SSCM arms)

\begin{tabular}{lrcc} 
Outcome & Estimate & $95 \% \mathrm{Cl}$ & $P$ \\
$\begin{array}{l}\text { Primary outcomes } \\
\quad\end{array}$ & & & \\
$\quad$ Body mass index & 1.32 & 0.76 to 1.88 & $<0.001$ \\
$\quad$ Weight & 3.55 & 2.07 to 5.03 & $<0.001$ \\
$\quad$ EDE Global & -1.29 & -1.81 to -0.78 & $<0.001$ \\
\hline $\begin{array}{l}\text { Secondary outcomes } \\
\quad \text { HADS anxiety }\end{array}$ & -2.50 & -4.50 to -0.50 & 0.014 \\
$\quad$ HADS depression & -3.09 & -4.87 to -1.32 & 0.001 \\
$\quad$ Clinical Impairment & -0.65 & -0.98 to -0.31 & $<0.001$ \\
Assessment & & & \\
$\quad$ Brixton Spacial & -2.14 & -4.57 to 0.29 & 0.084 \\
Anticipation Task & & & \\
Wisconsin Card & -6.63 & -16.54 to 3.29 & 0.189 \\
Sorting Task & & & \\
Trail Making Task & -4.91 & -10.70 to 0.88 & 0.096
\end{tabular}

MANTRA, Maudsley Model of Anorexia Nervosa Treatment for Adults; SSCM, specialist supportive clinical management; EDE Global, Eating Disorders Examination Global score; HADS, Hospital Anxiety and Depression Scale. as well as a novel theory-driven treatment. The strength of SSCM may lie in its simplicity, which allowed therapists to focus on facilitating a good working alliance while also requiring a focus on eating behaviours. However, in the earlier trial, advantages of SSCM disappeared over time. ${ }^{17}$ It will therefore be important to assess the longer-term effects of MANTRA and SSCM. One conclusion is that SSCM should be recommended for milder cases of anorexia where motivation for treatment is high.

\section{Strengths and limitations}

The strengths of the present study include that it tests an empirically based complex intervention developed for anorexia nervosa. The trial was conducted in a busy specialist eating disorder service, and so reflects real-life clinical practice. The sample size compares favourably to that of most other trials assessing adults with anorexia. We also ascertained that masking of assessors was successful, something many trials do not report. A novel feature is that like the patients, the therapists, too, were randomised. In previous trials, therapists were often asked to carry out more than one treatment. This can lead to contamination of treatments and increased cognitive load on therapists. Importantly, this is the first RCT of patients with anorexia nervosa to report use of neuropsychological measures as an outcome.

The study is limited by the fact that only some aspects of our maintenance model of anorexia nervosa were examined. For example, motivation was not examined, nor was any assessment of central coherence made, although this is thought to be an important component of the disorder's thinking style. Assessment of other aspects of the MANTRA model such as socio-emotional functioning was included and will be reported separately. A further limitation is the lack of a formal health economic analysis.

\section{Implications for future studies}

Since starting this trial, our understanding of what maintains anorexia nervosa has developed (e.g. Sternheim et al, ${ }^{39}$ Goddard et $a l^{40}$ ) and MANTRA has been refined. The revised version is being tested in larger trials which will allow subgroup analyses and include health economic assessments.

Ulrike Schmidt, MD, PhD, Anna Oldershaw, PhD, Section of Eating Disorders, King's College London, Institute of Psychiatry, London, UK; Fatima Jichi, MSC, Department of Biostatistics, King's College London, Institute of Psychiatry, London, UK; Lot Sternheim, MSC, Section of Eating Disorders, King's College London, Institute of Psychiatry, London, UK; Helen Startup, PhD, Eating Disorders Unit, South London and Maudsley NHS Foundation Trust, London, UK; Virginia McIntosh, PhD, Jennifer Jordan, PhD, University of Otago, Department of Psychological Medicine,

Christchurch, New Zealand; Kate Tchanturia, PhD, Section of Eating Disorders, King's College London, Institute of Psychiatry, London, UK; Geoffrey Wolff, MD, Michael Rooney, Eating Disorders Unit, South London and Maudsley NHS

Foundation Trust, London, UK; Sabine Landau, PhD, Department of Biostatistics, King's College London, Institute of Psychiatry, London, UK; Janet Treasure, MD, PhD, Section of Eating Disorders, King's College London, Institute of Psychiatry, London, UK

Correspondence: Professor Ulrike Schmidt, Section of Eating Disorders Unit (PO59), Institute of Psychiatry, De Crespigny Park, London SE5 8AF, UK. Email: ulrike.schmidt@kcl.ac.uk

First received 18 Mar 2012, final revision 25 May 2012, accepted 27 Jun 2012

\section{Funding}

This work was supported by the Psychiatry Research Trust, by a National Institute of Health Research (NIHR) Programme Grant for Applied Research (Reference number RP-PG-06061043) and by an NIHR methodology fellowship for F.J. The views expressed herein are those of the authors and not necessarily those of the NHS, NIHR or the Department of those
Health. 


\section{Acknowledgements}

We would like to thank the study therapists. We are grateful to Hannah de Jong and Martha Kenyon for their helpful comments on a draft of this paper.

\section{References}

1 Treasure J, Claudino AM, Zucker N. Eating disorders. Lancet 2010; 375 583-93.

2 Simon J, Schmidt U, Pilling S. The health service use and cost of eating disorders. Psychol Med 2005; 35: 1543-51.

3 Guillaume S, Jaussent I, Olié E, Genty C, Bringer J, Courtet P, et al. Characteristics of suicide attempts in anorexia and bulimia nervosa: a case-control study. PLOS One 2011; 6: e23578.

4 Roberts ME, Tchanturia K, Stahl D, Southgate L, Treasure J. A systematic review and meta-analysis of set-shifting ability in eating disorders. Psychol Med 2007; 37: 1075-84.

5 Lopez C, Tchanturia K, Stahl D, Treasure J. Central coherence in eating disorders: a systematic review. Psychol Med 2008; 38: 1393-404.

6 Oldershaw A, Hambrook D, Stahl D, Tchanturia K, Treasure J, Schmidt U. The socio-emotional processing stream in anorexia nervosa. Neurosci Biobehav Rev 2011; 35: 970-88.

7 National Institute for Health and Clinical Excellence. Eating Disorders: Core interventions in the Treatment and Management of Anorexia Nervosa, Bulimia Nervosa and Related Eating Disorders. British Psychological Society \& Gaskell, 2004

8 De Jong $\mathrm{H}$, Broadbent $\mathrm{H}$, Schmidt $\mathrm{U}$. A systematic review of dropout from treatment in outpatients with anorexia nervosa. Int J Eat Disord 2012; 45: 635-47.

9 Hall A, Crisp AH. Brief psychotherapy in the treatment of anorexia nervosa. Outcome at one year. Br J Psychiatry 1987; 151: 185-91.

10 Channon S, de Silva P, Hemsley D, Perkins R. A controlled trial of cognitive behavioural and behavioural treatment of anorexia nervosa. Behav Res Ther 1989; 27: 529-35.

11 Crisp AH, Norton K, Gowers S, Halek C, Bowyer C, Yeldham D, et al. A controlled study of the effect of therapies aimed at adolescent and family psychopathology in anorexia nervosa. Br J Psychiatry 1991; 159: 325-33.

12 Treasure J, Todd G, Brolly M, Tiller J, Nehmed A, Denman F. A pilot study of a randomised trial of cognitive analytical therapy vs educational behavioural therapy for adult anorexia nervosa. Behav Res Ther 1995; 33: 363-7.

13 Bachar E, Latzer Y, Kreitler S, Berry EM. Empirical comparison of two psychological therapies. Self psychology and cognitive orientation in the treatment of anorexia and bulimia. J Psychother Pract Res 1999; 8: 115-28.

14 Serfaty MA, Turkington D, Heap M, Ledsham L, Jolley E. Cognitive therapy vs dietary counseling in the outpatient treatment of anorexia nervosa: effects of the treatment phase. Eur Eat Disord Rev 1999; 7: 334-350.

15 Dare C, Eisler I, Russell G, Treasure J, Dodge L. Psychological therapies for adults with anorexia nervosa: randomised controlled trial of out-patient treatments. Br J Psychiatry 2001; 178: 216-21.

16 McIntosh VV, Jordan J, Carter FA, Luty SE, McKenzie JM, Bulik CM, et al. Three psychotherapies for anorexia nervosa: a randomized, controlled trial. Am J Psychiatry 2005; 162: 741-7.

17 Carter FA, Jordan J, McIntosh VV, Luty SE, McKenzie JM, Frampton CM, et al. The long-term efficacy of three psychotherapies for anorexia nervosa: a randomized, controlled trial. Int J Eat Disord 2011; 44: 647-54.

18 Schmidt U, Treasure J. Anorexia nervosa: valued and visible. A cognitiveinterpersonal maintenance model and its implications for research and practice. Br J Clin Psychol 2006; 45 (Pt 3): 343-66.

19 Wade TD, Treasure J, Schmidt U. A case series evaluation of the Maudsley Model for treatment of adults with anorexia nervosa. Eur Eat Disord Rev 2011; 19: 382-9.
20 Thomas JJ, Vartanian LR, Brownell KD. The relationship between eating disorder not otherwise specified (EDNOS) and officially recognized eating disorders: meta-analysis and implications for DSM. Psychol Bull 2009; 135 407-33

21 Bergh C, Brodin U, Lindberg G, Södersten P. Randomized controlled trial of a treatment for anorexia and bulimia nervosa. Proc Natl Acad Sci USA 2002; 99: 9486-91.

22 Serpell L, Treasure J, Teasdale J, Sullivan V. Anorexia nervosa: friend or foe? Int J Eat Disord 1999; 25: 177-86.

23 Kyriacou O, Treasure J, Schmidt U. Expressed emotion in eating disorders assessed via self-report: an examination of factors associated with expressed emotion in carers of people with anorexia nervosa in comparison to control families. Int J Eat Disord 2008; 41: 37-46.

24 Sepulveda AR, Kyriacou O, Treasure J. Development and validation of the accommodation and enabling scale for eating disorders (AESED) for caregivers in eating disorders. BMC Health Serv Res 2009; 9: 171.

25 Miller WR, Rollnick S. Motivational Interviewing. Preparing People for Change (2nd edn). Guilford Press, 2002.

26 National Institute for Health and Clinical Excellence. Behaviour Change at Population, Community and Individual Levels. British Psychological Society \& Gaskell, 2007

27 McIntosh VV, Jordan J, Luty SE, Carter FA, McKenzie JM, Bulik CM, et al Specialist supportive clinical management for anorexia nervosa. Int J Eat Disord 2006; 39: 625-32.

28 Fairburn CG, Cooper Z, O'Connor M. Eating disorder examination (Edition 16.0D). In Cognitive Behavior Therapy and Eating Disorders (ed. CG Fairburn): 265-308. Guilford Press, 2008.

29 Bohn K, Fairburn CG. The Clinical Impairment Assessment questionnaire (CIA 3.0). In Cognitive Behavioral Therapy for Eating Disorders (ed. CG Fairburn): 315-8. Guildford Press, 2008.

30 Zigmond AS, Snaith RP. The Hospital Anxiety and Depression Scale, Acta Psychiatr Scand 1983; 67: 361-70.

31 Burgess PW, Challice T. The Hayling and Brixton Tests. Thames Valley Test Company, 1997

32 Heaton RK, Chelune GJ, Talley JL, Kay G, Curtiss G. Wisconsin Card Sorting Test. Computer Version 4 (WCST:CV4). Psychological Assessment Resources, 1993.

33 Kravariti E, Morris RG, Rabe-Hesketh S, Murray RM, Frangou S. The Maudsley Early-Onset Schizophrenia Study: cognitive function in adolescent-onset schizophrenia. Schizophr Res 2003; 65: 95-103.

34 Schafer JL. Multiple imputation: a primer. Stat Methods Med Res 1999; 8: 3-15.

35 Royston P. Multiple imputation of missing values: further update of ice, with an emphasis on categorical variables. Stata J 2009; 9: 466-77.

36 Fairburn CG, Cooper Z, Doll HA, O'Connor ME, Bohn K, Hawker DM, et al. Transdiagnostic cognitive-behavioral therapy for patients with eating disorders: a two-site trial with 60-week follow-up. Am J Psychiatry 2009; 166: 311-9.

37 Couturier J, Lock J. What is recovery in adolescent anorexia nervosa? Int J Eat Disord 2006; 39: 550-5.

38 Long CG, Fitzgerald KA, Hollin CR. Treatment of chronic anorexia nervosa: a 4-year follow-up of adult patients treated in an acute inpatient setting. Clin Psychol Psychother 2012; 19: 1-13.

39 Sternheim L, Startup H, Schmidt U. An experimental exploration of behavioral and cognitive-emotional aspects of intolerance of uncertainty in eating disorder patients. J Anxiety Disord 2011; 25: 806-12.

40 Goddard E, Macdonald P, Sepulveda AR, Naumann U, Landau S, Schmidt U, et al. Cognitive interpersonal maintenance model of eating disorders: intervention for carers. Br J Psychiatry 2011; 199: 225-31. 